\title{
Automated Image Analysis Method for p-vivax Malaria Parasite Detection in Thick Film Blood Images
} Método automatizado de análisis de imágenes para detección del parásito de la malaria p-vivax en imágenes de gota gruesa

Leonardo Yunda, Ph.D(c)

leyunda@ieee.org

Universidad Santiago de Cali, Colombia

Andrés Alarcón Ramirez, M.Sc alarconandres2001@gmail.com Laboratory of Applied Remote Sensing and Image Processing Universidad de Puerto Rico, Recinto Mayagüez

Jorge Millán, PhD jmillan@sigmabiomedical.com Sigma Biomedical, Hialeah, FL

Fecha de recepción: Febrero 12 de 2012 Fecha de aceptación: Marzo 20 de 2012

\section{Keywords}

Malaria; Thick film microscopy;

Neuronal networks; principal component analysis.

\section{Palabras clave}

Malaria; gota gruesa; microscopia; redes neuronales; Análisis de Componentes Principales; PCA.

Colciencias tipo

\section{Abstract}

An image analysis method for Malaria parasite detection in thick film blood images is described. The developed method uses a combination of AGNES [Absence of gradients and Nernstian equilibrium stripping] and Morphological Gradient techniques in the image segmentation stage. Wavelet-based feature extraction is followed by a neural network classification stage. Principal Component Analysis (PCA) is used to reduce the number of features and improve the performance of the neuronal network. The true positive rate for determining a specific parasite was of $77.19 \%$, while a $76.45 \%$ was obtained in determining at least a parasite in a microscopy image.

\section{Resumen}

El artículo describe un método de análisis de gota gruesa para la detección del parásito de la malaria en la sangre, realizado a partir del análisis de imágenes. Para la etapa de segmentación de las imágenes el método desarrollado combina las técnicas AGNES [Absence of gradients and Nernstian equilibrium stripping] y del Gradiente Morfológico. La extracción de características se basa en la transformada de Wavelet y es seguida por una etapa de clasificación de la red neuronal. El método utiliza la técnica de Análisis de Componentes Principales (PCA) para reducir el número de funciones y mejorar el rendimiento de la red neuronal. La tasa de detección efectiva (True-Positive rate) lograda fue de $77,19 \%$ en la determinación de un parásito específico, y de 76,45\% en la determinación de al menos un parásito en una imagen de microscopio. 


\section{Introduction}

Malaria is the world's most notorious tropical parasitic disease, and kills more people than any other contagious disease except Tuberculosis (TB). Malaria is responsible for more than 300 million clinical cases and between one and three million deaths occurring in the world each year (Gutiérrez \& Arróspide, 2003). The principal population at risk includes mainly children under five years and pregnant women from endemic areas, as well as nonimmune visitors to those areas. Fifty years after the beginning of the worldwide Malaria Eradication Program, malaria continues to increase and the control tools designed for this program have become less effective due to drug and insecticide resistance developed by parasites and mosquito vectors, respectively. This deteriorating situation of malaria control has stimulated a search for rapid, sensitive, specific and affordable diagnostic tools for Malaria detection so effective programs can be implemented for the treatment of this potentially fatal parasitic disease.

Conventional and widely-used malaria parasite detection methods use microscopic examination of Giemsa-stained thick and thin blood smears (Hanscheid, 2003). In these, examination of blood films requires the availability of qualified and experienced personnel, which is not readily available in endemic areas, for the recognition of malaria parasites. Visual parasite recognition is also a routine and time-consuming task and prone to misdiagnosis. PCR (Polymerase Chain Reaction) offers an alternative to microscopy which has shown in many cases to have superior sensitivity and specificity (Mens, Spieker, Omar, Heijnen, Schallig, \& Kager, 2007). However, cost makes it prohibitive for widespread use in malaria endemic areas, which are generally associated with poverty and low economic development. Recently, rapid methods such as Rapid Diagnostic Tests (RDTs) (Moody, 2002) have shown high accuracy in laboratory and field-based studies, though their sensitivity declines at low parasitemias (< 300-500/_L) (Iqbal, Khalid, \& Hira, 2002; Murray, Bell, Gasser, \& Wongsrichanalai, 2003). Cost may still be a constraint for widespread use.

Several image processing methods have been reported for the computerized recognition of White Blood Cells (WBC) in color microscopy images of blood (Katz, 2000). There exists applications for blood cell counting in digital images using the Watershed method (Angulo, 2003 ; Wu, Gauthier, \& Levine, 1995; Yang, Meer, \& Foran, 2002), using morphological, color and texture features in the object extraction process. One of the reported methods (Katz, 2000) uses a Canny edge detection filter (Canny, 1986) for border identification. The extraction stage uses geometric characteristics such as the borders of objects of interests, and color characteristics that use the difference between each of the RGB color components and the maximum and minimum values of the pixels within the object. The classification stage employs a MLP neural network with a hidden layer. This 
method was then compared to a Bayesian classifier resulting in better performance of the neural network method.

Computerized malaria parasite recognition has been reported using color image thresholding methods (di Ruberto, Dempster, Khan, \& Jarra, 2000, 2002; Rao, 2004; Sio et al., 2007). Images were pre-processed to remove the illumination bias which was estimated as the difference between the histogram peak positions of the color channels (i.e. peak of blue - peak red/green). The markers for the parasites were extracted using a threshold at the halfway point of the cumulative histogram. However, in a diagnosis scenario, for every ample, such an approach would fail by marking all white blood cells (WBC), platelets and artifacts as parasites. The work of Pinzón, Garavito, Hata, Arteaga y García (2004) describes recognition of annular forms in thick blood films using a variant of the Hough transform. In this method, circular objects are identified, and nonlinear morphological filters are applied to enhance circular shaped parasites. Reported results show detected erythrocytes of circular shape and uniform size, however it does not determine if recognized circular objects correspond to malaria parasites. The work by Premaratnea, Dharshani, Shyam, Pererab and Rajapakshab (2007) explores detection of rings in a peripheral smear image using a MLP neural network with a hidden layer using as input a fraction of the original image which has been previously marked by the user. The work by Le, Bretschneider, Kuss and Preiser (2008) describes a semi automated image processing method for detection of plasmodium falciparum parasites in Giemsa-stained blood smear images. The described method is limited by the required prior knowledge of the erythrocyte area information which is needed for the parasitaemia calculation. A recent review of computer vision methods for microscopy diagnosis of malaria is given by Tek, Dempster and Kale (2009).

The present work explores a new image analysis method for the detection and classification of Plasmodium falciparum gametocytes, as well as esquizontes and gametocytes of Plasmodium vivax parasite images. The proposed processing method uses digital color images of thick blood films obtained from a digital camera coupled to a trinocular microscope (Romero \& Sarmiento, 2004). The method uses the K-NN technique for the detection of gametocytes in color images, achieving good results on a small number of input images.

\section{Acquisition and visualization system}

Blood smear malaria images are acquired and digitized using a microscope image acquisition and visualization system composed of:

» microscope Axiostar plus,

» adapter Triocular Carl Zeiss,

» digital camera Canon PowerShot G6 de 7.1 MP,

» personal computer, and

» software interface for digital image capture, visualization and image analysis. 
The digital camera is coupled to the Axiostar microscope by the triocular adapter which is mounted on the microscope for the digital image capture of the slides. Inmesion lens with $100 \mathrm{X}$ magnification were used. The triocular adaptor has a magnification stage of 10 , which produces a total combined magnification of $1000 \mathrm{X}$ seen by the digital camera.

\section{Image selection}

Using the image acquisition system, 20 thick blood smear slides were used for image acquisition, five of which were recognized as positives with plasmodium falciparum, 8 identified positive with plasmodium vivax and 7 were identified as not having the parasite. From the slides, the acquisition system captured 248 images. From these, 100 were identified and labeled as negative images, i.e., no presence of any parasite form, 78 images were identified and labeled as having plasmodium falciparum of gametocyte type, 38 images with plasmodium vivax of gametocyte type, and 32 images with plasmodium vivax of esquizonte type. From the labeled images, a total of 163 forms of plasmodium vivax, gametocyte type, were obtained; 113 forms of plasmodium vivax of esquizonte type, and 78 forms of plasmodium falciparum, gametocyte type.

From all of the forms of plasmodium vivax, gametocyte type, 85 were randomly selected to conform the training group. The rest of the images were used in the classification process. Similarly, for the plasmodium vivax, esquizonte type, 62 forms were selected for the training group, and 51 images for the classification stage. For plasmodium falciparum, gametocyte type, 42 forms for the training stage, and 36 for the classification stage. In the other hand, 171 forms of sediments (artifacts resulting from the slide stain process) present in the group of captured images were used to form the training set for the forms that do not belong to any of the classes and parasite forms studied in this work. The training set also consisted of 46 forms of red blood cells present in the group of captured images.

\section{Segmentation stage}

The segmentation stage used a combination of image processing methods for border and region detection that allow rejection of the image background identifying each of the objects present in the processed image. Initially, the morphological gradient method is used to enhance the borders of the objects present in the image. This is followed by a threshold detection stage using the K-Media method.

The pixels identified as borders between the objects and the backgrounds in the last processing stage are assigned to each of the existing regions. For this, the Laplacian operator was used to identify which pixels are parts of the interior or exterior of the objects present in the image. Applying the logical operators AND and NOT en each of the images, a three level image is generated, which corresponds to the first division of pixels that belong to the background and the object, which are also part of the background and the objects, and that are located in zones where big changes in intensity occur. The remaining region is not assigned to one of the processed groups as this requires an additional processing method for its determination. 
In the other hand, to improve the initial conditions of the next segmentation stage, those pixels that were recovered using the morphological gradient and that did not make part of any object in the input image were eliminated. To accomplish this, an erosion operation (Angulo \& Serra, 2005) is performed, followed by two dilations (Angulo \& Serra, 2005; Kim \& Romeike, 2006) in the image generated from the morphological gradient operation. A logical operation AND is performed between the output image of the morphological operation with the input image. This way, the remaining pixels generated from the dilation operation are discarded.

The result of the erosion and two dilation operations on the input image allows discarding those pixels that constitute subtle borders in the original image and that do not correspond to borders between the objects and their background. The dilation operations reconstruct the original borders that are part of the input image.

The AGNES (Getz \& Levine, 2000; Van der Laan \& Pollard, 2003) and K-Median techniques were used to assign the remaining number of pixels to each region, using as the starting point the image regions previously identified as objects and background. AGNES and the K-Median were locally applied on rectangular sectors (masks) in which the image was divided. The size of the mask was $20 \%$ of the input image. This value was defined experimentally. In those cases were the mask is very small, it is seen a square effect which enhances the limits in which the image is divided. Similarly for higher values, the output images show non uniform stains, caused by the wrong assignment of pixels belonging to the background of the input images as objects of the output image.

The next processing step consists in the elimination of objects that are not part of the original image and that have been wrongly generated by the segmentation algorithm. For this, a comparison is performed between the number of pixels that are part of the border of each object identified in the output image and the number pixels present in the binary image obtained with the morphological gradient and the K-median operators. Thus, if the difference between those values is not below a selected threshold, the object is eliminated. The last step consists in reapplying the erosion process followed by two dilations and a logical AND operation on the output image to eliminate objects of reduced size (less than five pixels). The processing results obtained with a set of input images are shown in Figure 1.

Figure 1 shows the developed segmentation method being applied on a set of input images. The proposed algorithm is suitable for segmentation of thick smear blood images since it is capable of extracting the background from the objects, independently of color variations that can occur as a result of coloring of the microscope slides where the images are taken from.

\section{Object detection on segmented images}

After the segmentation stage is completed, each of the elements that constitute the associated region with each object is detected. For this, a set of pixels from the output 

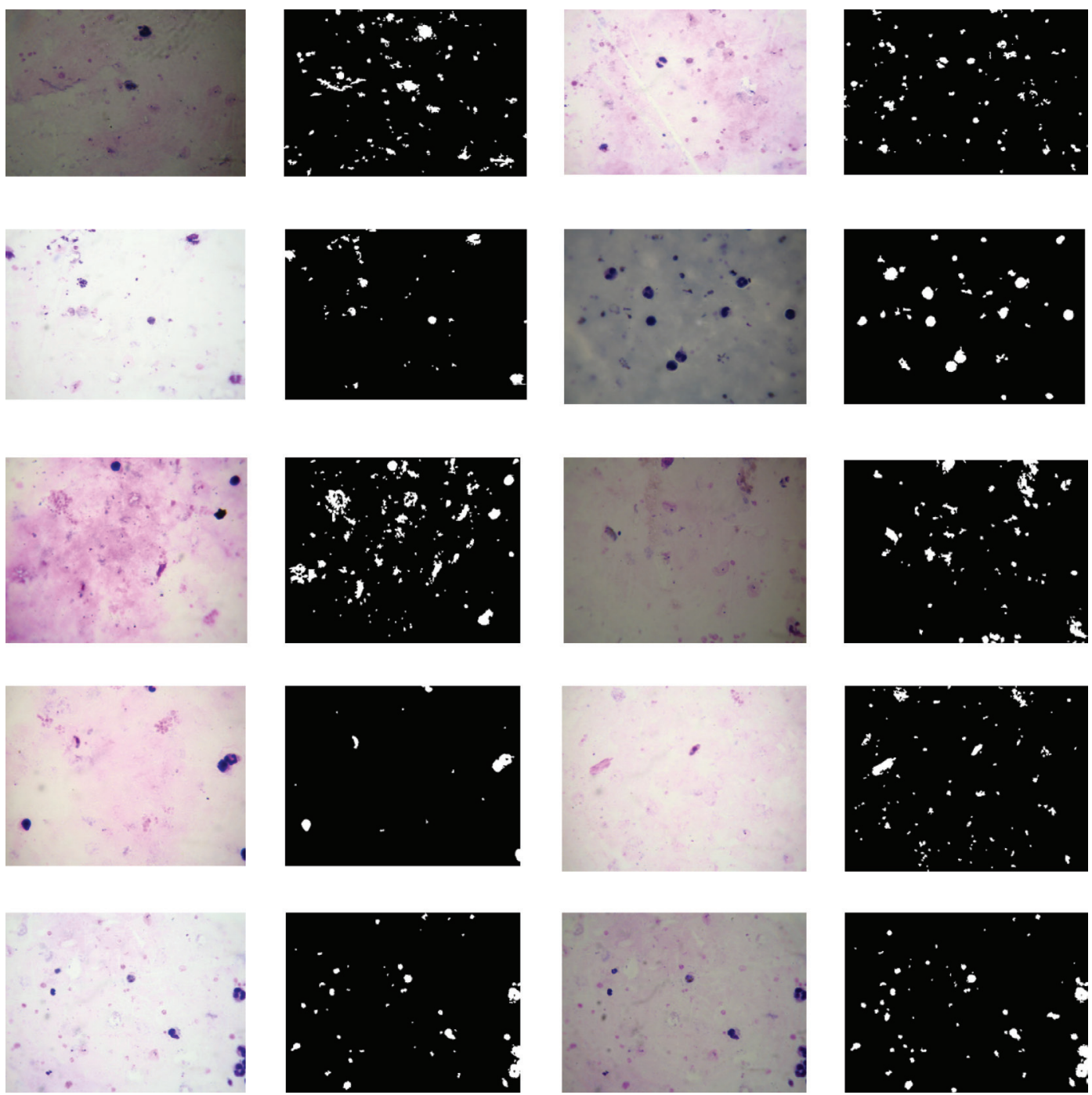

Figure 1. Segmentation operator results on a set of thick blood smear input images

binary image is randomly taken from the total set of pixels that were classified as objects in the segmentation stage. This constitutes the starting point to find the particular element that includes the selected pixel. From this, a dilation process is performed followed by an AND logical operation. The first process is in charge of increasing as much as possible the number of pixels that include the new region, whereas the second step makes sure that the object shape is preserved. This process is repeated until the element to classify does not go through further changes. When this point is reached, the pixels that belong to a particular element are associated, and then the process is followed with the remaining pixels that were associated in the segmentation stage as objects. 
At the end of the process, there will be as many classes as particular objects that are found in the original image; each of them associated with a set of pixels that correspond to them.

Results of applying this object detection algorithm in this processing stage on a set of input images are shown in Figure 2.

\section{A. Color Features}

The captured color images are composed of three color components: Red, Green and Blue, for each of the pixels in the images. Hence, for each of the objects extracted in the
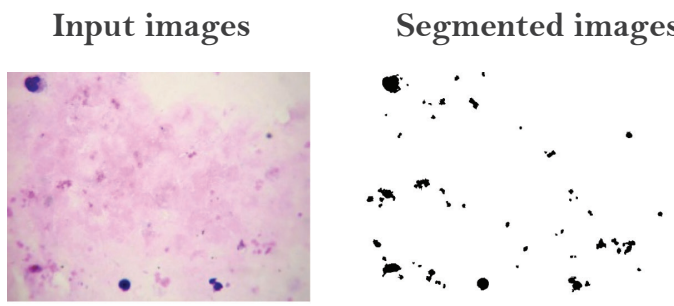

Border detection
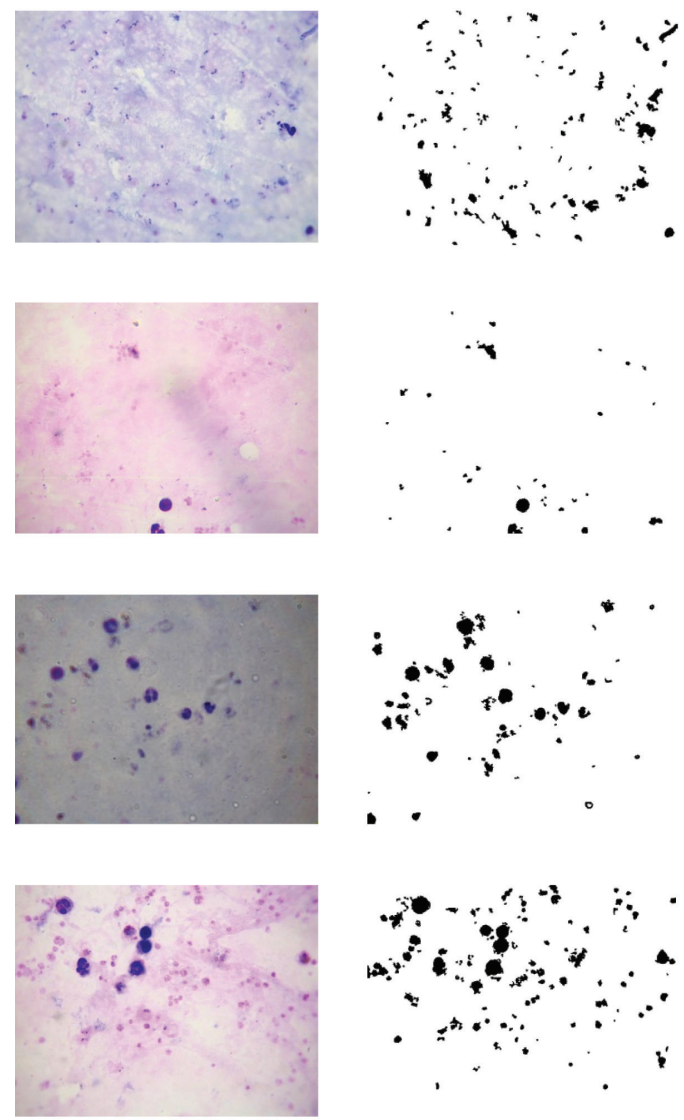

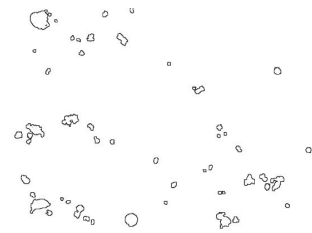

Object detection
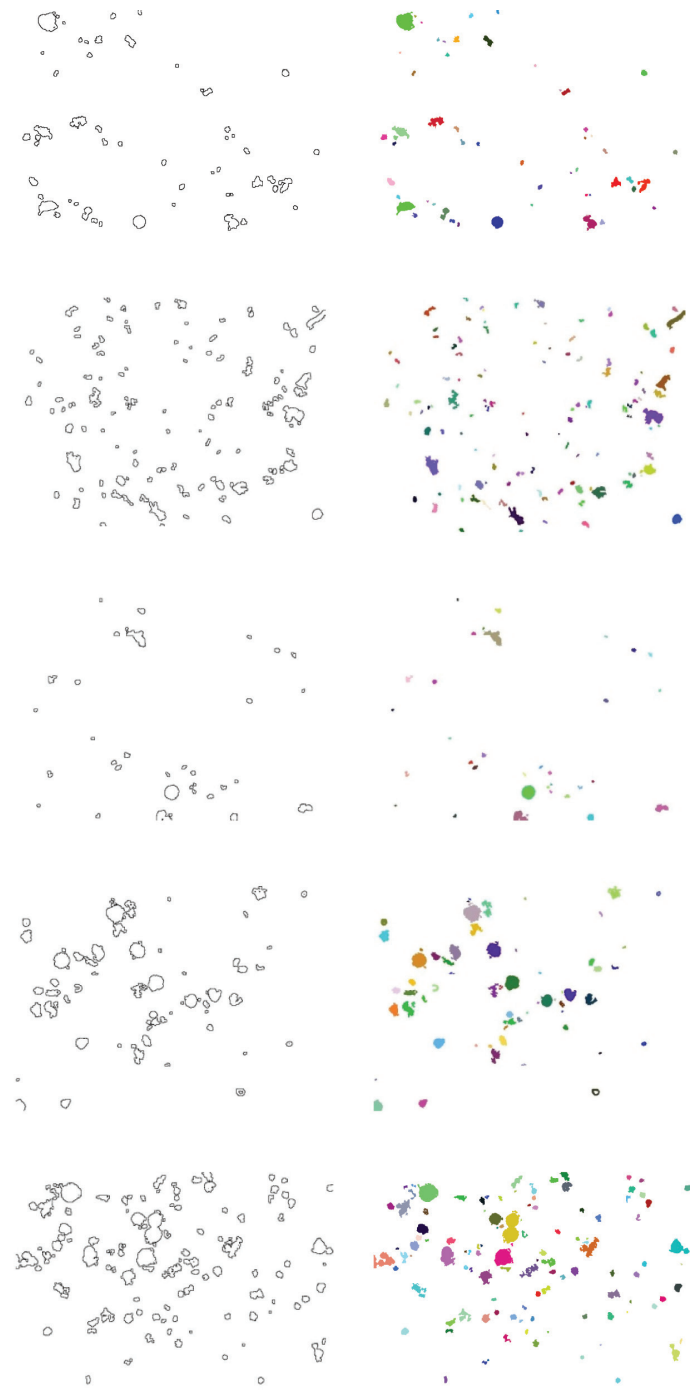

Figure 2. Segmentation and object detection on a set of thick blood smear images 
segmentation stage, their characteristics and features need to be calculated in each of the color components present in each of the image planes.

Each of the malaria parasites features a particular color tone, which is dependent on the coloring of each of the thick blood smear images. Thus, a set of descriptors must be selected that not only allow extracting of information related to color, but consider the variations that exist between each of the elements that constitute the existing objects. The color characteristics considered in this work are:

» standard deviation,

» 7 Hough moments of color, and

» color range

Thus, there are nine descriptors considered in this work for each the three color components, for a total of 27 total characteristics used.

\section{B. Texture Characteristics}

This work explored two methods to extract color characteristics of an object. The first method starts from a co occurrence matrix, and the second method is realized using the Wavelet transform. The co occurrence matrix is calculated for 4 distinct directions, with a distance between pixels of 1 . This generates 12 matrices of which 4 correspond to each direction, with each of these matrices having three matrices on each color component. From each matrix, the following descriptors are calculated

» homogeneity,

» contrast,

» GLMSR (General Linear Model Simple Regression)

» standard deviation,

» second angular moment, and

» correlation

There are a total of 72 calculated characteristics.

The discrete Wavelet transform is applied to each region of interest in the input image with two decomposition levels, through the combined action of low pass and high pass filters in each of the columns and rows of the image. With this, 6 new images are generated, and the following descriptors are calculated:

» Energy

» Mean

»Standard deviation

As each generated image has three components, the total number of descriptors increases to 54 .

The Wavelet families used in the processing of the Wavelet transform are:

»Haar;

» Biortogonal 1,3; 
»Daubichies 2; and

»Daubichies 8.

With the above, for each object of interest identified in the segmentation stage, 11 shape descriptors, 27 color descriptors, and 72 texture descriptors are calculated for the co occurrence matrix or 54 for the Wavelet transform.

The texture features resulting from the co occurrence matrix and from the Wavelet transform are not used together since it will considerably increase the total number of descriptors that are calculated for each object. Hence, the total number of descriptors is 110 when the co occurrence matrix is used and 92 when the Wavelet transform is used.

Besides calculating the features vector for each of the extracted objects in the segmentation stage, it is necessary to arrange a set of feature vectors for each of the existent object classes so a database is conformed. From this database, the various processing methods used in the classification stage can use this information to associate an object to a particular class.

Due to the large number of descriptors considered in this work, it is necessary to use a variable reduction method that is in charge in identifying those features that are redundant and do not carry new information. The Principal Component Analysis (PCA) is a useful method to accomplish variable reduction. PCA is then applied on the set of features such as shape, color and texture, previously stored in the database.

With the total set of features, it is found that the first three components explain $87.3 \%$ of the total variance; hence a good approximation can be achieved analyzing only these components in the correlation circle as shown in Figure 3.

Those features that are closely located in each of the correlation circles contribute with redundant information; hence it is necessary to keep only one of those features. With this, the number of features is reduced from 110 to 34 , when the co occurrence matrix is used and from 92 to 49 when the Wavelet transform is used.

\section{Classification stage}

In this stage, a multilayer neural network of the Perceptrom type was used. This neural network is in charge of classifying gametocytes of plasmodium falciparum, and esquizontes and gametocytes of the plasmodium vivax type.

The neural network architecture is characterized by an input layer, one or two hidden layers and an output layer. The neural network has 5 neurons in the output later, of which three represent the parasites of interest, and the two remaining correspond to sediments and white blood cells.

The training algorithms used in the learning stage for each of the neural networks are the following:

» Traingd,

» Traingda, 
Sedimentation Diagram

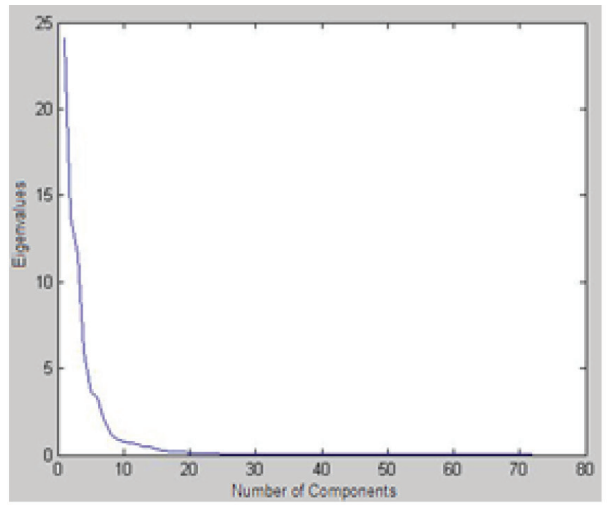

Correlation circle 1-3 components

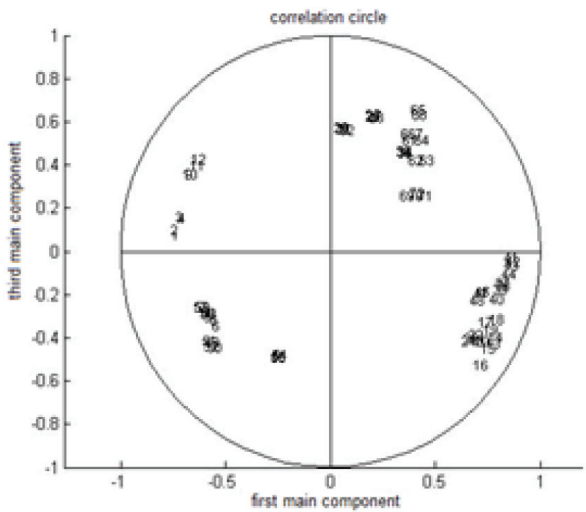

Correlation Circle 1-2 components

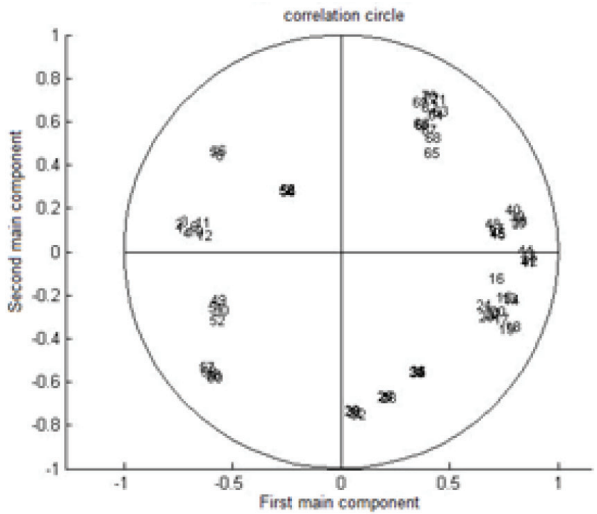

Correlation circle 2-3 component

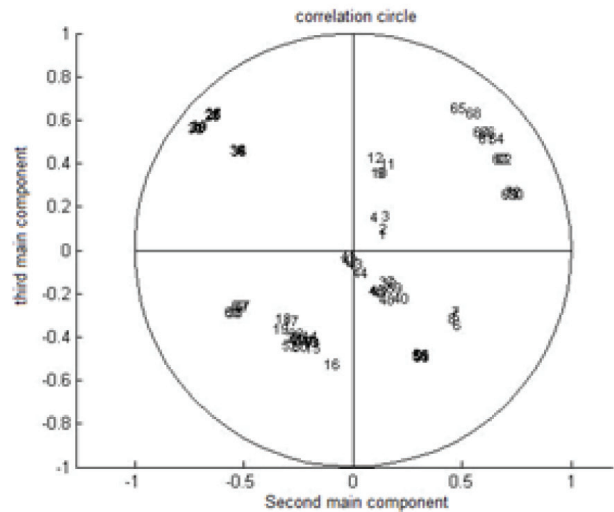

Figure 3. Principal Component Analysis

» Traingda,

» Traingdm,

» Traingda, and

»Trainscg.

To validate the existing neural networks, the following input images are used: 36 images with plasmodium falciparum gametocytes, 20 with Esquizontes of plasmodium vivax type, 19 with gametocytes of plasmodium vivax and 35 images that do not contain any of the parasite types studied in this work.

Each of the images identified as malaria positive may contain one or two parasites of the same type and class, which increases the total amount of available parasites, as illustrated in Table 1. 
Table 1. Malaria parasites in the input images

\begin{tabular}{ll}
\hline \hline Type of Parasites & $\#$ \\
\hline \hline Gametocytes (falciparum) & 36 \\
\hline Gametocytes (vivax) & 85 \\
\hline Esquizontes (vivax) & 51 \\
\hline \hline
\end{tabular}

Two distinct methods were explored to measure the percentage of successes that the neural network obtains in the classification of the parasite types considered in this work. The first measure is sensitivity, which measures the system's capacity to recognize at least one parasite in the thick blood smear image, without considering the type, class or the number of parasites present in the image. The second method is specificity, which takes into account the type, class and the number of parasites present in the input image.

Figure 4 shows the percentage of successes for the specificity analysis of the three neural networks that provided the best results.

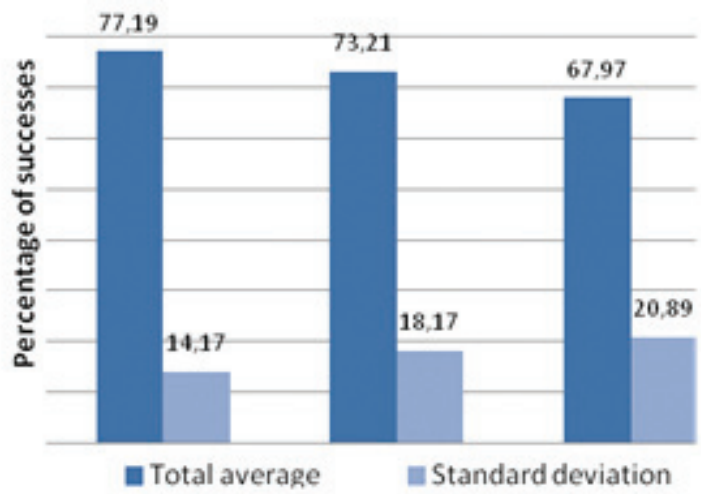

Figure 4. Success percentages for the three neural networks that achieved the best specificity results

The highest percentage in the classification of the three types of parasites and the sediment class (specificity analysis) was $77.19 \%$ with a Standard deviation of 14.17 . This was obtained with the neural network that was trained using the Trainscg algorithm, which incorporated texture features of the Wavelets Db2 family, which had 17 and 10 neurons in the first and second hidden layer, respectively.

The classification percentages obtained by the three neural networks for each of the classes studied in this project are shown in Figure 5. 


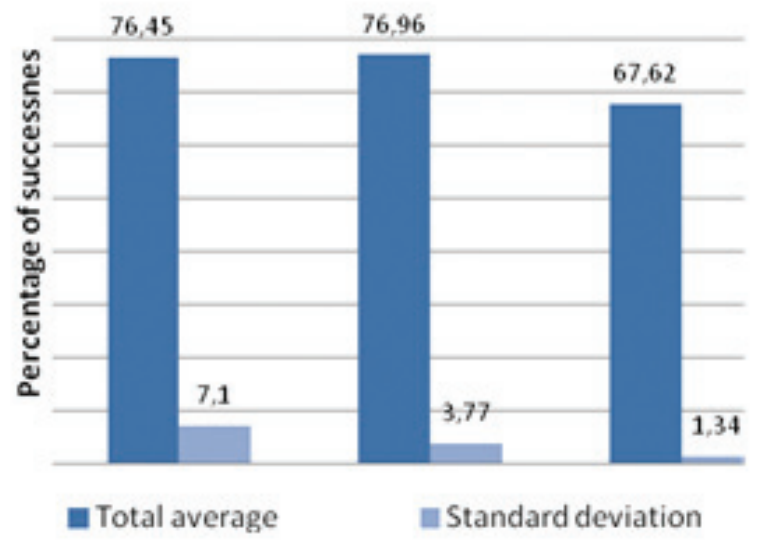

Figure 5. Success percentages for each of the parasite classes and sediments obtained by each of the three neural networks that provided the best results in the specificity analysis

In the last figure it is observed that the highest classification percentage obtained for the plasmodium falciparum parasite type was $75 \%$, which was obtained with the neural network that had the second best classification score. For the plasmodium vivax case of gametocyte and esquizonte types, the classification percentages were $71.6 \%$ and $66.7 \%$, respectively. These were obtained with the neural network that had the best global performance. For the sediment class, it was achieved a percentage of $98.24 \%$, with the network that had the second best performance.

Figure 6 shows the highest three percentages obtained in the sensitivity analysis.

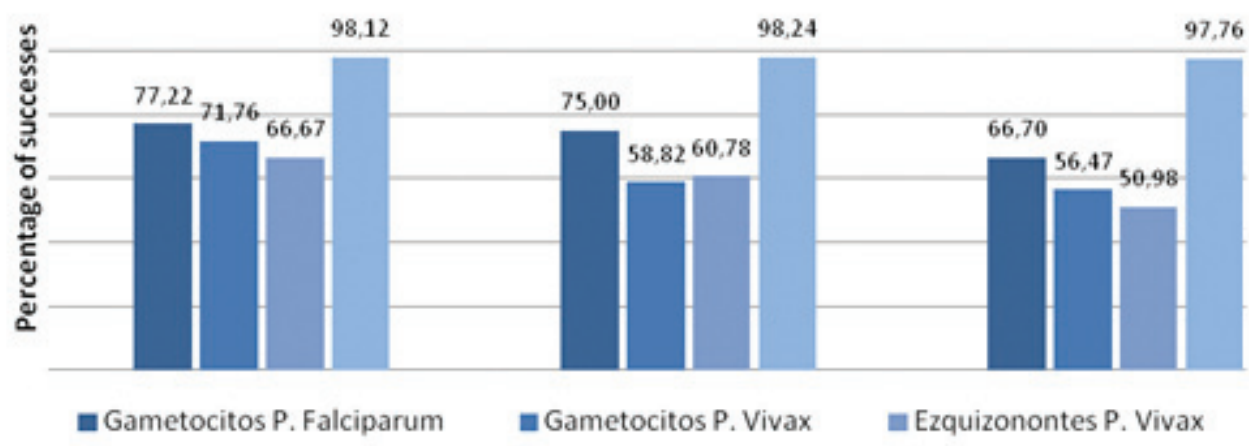

Figure 6. Success percentages for the three neural networks that achieved the best sensitivity results 
From Figure 6, it is seen that the highest classification percentage when determining if an image had at least one of the parasite types was $76.9 \%$. This result was obtained with the neural network that was trained with the Trainscg algorithm, which incorporated texture features, extracted from the Wavelet family Bior 1.3, with 14 and 10 neurons in the first and second hidden layer, respectively.

Figure 7 shows the classification percentages for the positive and negative images obtained with the three neural networks of best performance in the sensitivity analysis

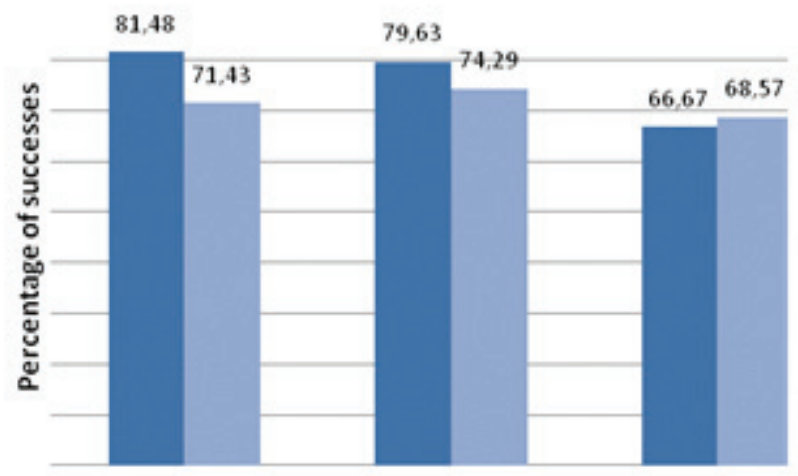

nositive images negative images

Figure 7. Success percentages for the three neural networks that achieved the best performance in the sensitivity analysis

From Figure 7 it is observed that the highest percentage of identification for at least one type of malaria parasite was $81.4 \%$, which was achieved with the neural network that had the second best performance in the sensitivity analysis. The identification of an image as a negative (no malaria parasites found) achieved a success percentage of $74.29 \%$. The results from the sensitivity and specificity analysis indicate that the neural networks with two hidden layers obtained better identification performance than the neural networks with only one hidden layer.

\section{Conclusions}

The results obtained with neural networks of one hidden layer, both in sensitivity as well as in specificity, were not as good as those results obtained using neural networks of two hidden layers. This is understandable due to the complexity of the system which makes difficult to separate the various classes in a single surface. 
The implementation of the method focused on border and region detection, did not provide good results for the segmentation of thick blood smear images. However, when various techniques were combined, an algorithm was generated that was robust enough to identify the objects and background present in the image.

The results achieved by the developed method were closely related to the capture and use of thick blood images in good conditions, where it was clearly determined the presence or not presence of the distinct forms of parasites that cause Malaria. In those images the background was uniform and exempt from granulates caused by the staining process in the microscopic slides. SAT

\section{References}

Rao, K (2004). Application of mathematical morphology to biomedical image processing (Ph.D. thesis). Westminster University, London, UK.

Moody, A (2002). Rapid diagnostic tests for malaria parasites. Clinical Microbiology Revieres 15(1), 66-78.

Katz, A. (2000). Image analysis and supervised learning in the automated differentiation of white blood cells from microscopic images (Master's thesis). RMIT University, Melbourne, Australia.

Ruberto, C., Dempster, A., Khan, S., \& Jarra, B. (2002). Analysis of infected blood cell images using morphological operators. Image and Vision Computing, 20(2),133-146.

Ruberto, C., Dempster, A., Khan, S., \& Jarra, B. (2000). Automatic thresholding of infected blood images using granulometry and regional extrema. In Proceedings, 15th. International Conference on Pattern Recognition, pp.3445-3448.

Canny, J. (1986). A computational approach to edge detection. IEEE Transactions on Pattern Analysis and Machine Intelligence, 8(6), 679-698

Tek, F.B., Dempster, A., \& Kale, I. (2009, July). Computer vision for microscopy diagnosis of malaria. Malaria Journal, 8. doi:10.1186/1475-2875-8-153

Getz G. \& Levine E. (2000). Coupled two-way clustering analysis of gene microarray data. Proceedings of the National Academy of sciences of the United States of America, 97(22), 12079.12084.

Gutiérrez, S., \& Arróspide, N. (2003). Manual de procedimientos de laboratorio para el diagnostico de Malaria (Serie de Normas Tecnicas N 39). Lima, Perú: Instituto Nacional de Salud.

Hanscheid, T. 2003. Current strategies to avoid misdiagnosis of malaria. Clinical Microbiology and Infection, 9(6), 497504.

Iqbal J, Khalid N, \& Hira P.R. (2002), Comparison of two commercial assays with expert microscopy for 
confirmation of symptomatically diagnosed malaria. Journal of Clinical Microbiology, 4O(12), 4675-4678

Angulo, J. (2003). Morphologie mathématique et indexation d'images couleur. Application à la microscopie en biomédecine (Doctoral Thesis), University of Minas, Paris, Francia.

Angulo, J. \& Serra, J. (2005). Segmentación de imágenes en color utilizando histogramas bi-variables en espacios de color polares luminancia/saturación/ matiz. Paris, France: Centro de Morfología Matemática.

Wu, K., Gauthier, D., \&Levine, M.D. (1995). Live cell image segmentation. IEEE Transactions on Biomedical Engineering, 42(1), 1-12.

Yang, L., Meer, P., \& Foran, D. (2005). Unsupervised segmentation based on robust estimation and color active contour models. IEEE Transactions on Information Techonology in Biomedicine, 9(3), 475-486

Mens, P., Spieker, N., Omar, S., Heijnen, M., Schallig, H., \& Kager P.A. (2007). Is molecular biology the best alternative for diagnosis of malaria to microscopy? A comparison between microscopy, antigen detection and molecular tests in rural Kenya and urban Tanzania. Tropical Medicine E' International Health, $12(2), 238-244$.

Le, M.T., Bretschneider, T., Kuss, C \& Preiser, P. (2008, march). A novel semiautomatic image processing approach to determine Plasmodium falciparum parasitemia in Giemsa-stained thin blood smears", BMC Cell Biology,

\section{9(art.15).}

Murray, C.K., Bell, D., Gasser, R.A., \& Wongsrichanalai, C. (2003). Rapid diagnostic testing for malaria. Tropical Medicine \& International Health, 8(10): 876-883.

Roberto Pinzón , Giovanny Garavito , Yoshie Hata, Lucía Arteaga , Juan David García, "Desarrollo de un Sistema de Análisis Automático de Imágenes de Extendidos Sanguíneos” Memorias del Congreso Espanol de la Sociedad de Ingeniería Biomédica, 2004:45-59

Romero, E., \& Sarmiento, W.J (2004). Automatic detection of malaria parasites in thick blood films stained with Haematoxylin-Eosin (presentado en III Iberian Latin American and Caribbean congress of Medical Physics, ALFIM 2004). Rio de Janeiro, Brazil.

Sio, S., Sun, W., Kumar, S., Bin, W., Tan, S., Ong, S., Kikuchi, H., Oshima, Y., \& Tan, K. (2007). Malaria count: an image analysis-based program for the accurate determination of parasitemia. Journal of Microbiological Methods 68 (1), 11-18.

Premaratnea, S., Dharshani, N., Shyam, F., Pererab, W., \& Rajapakshab, A. (2007). A neural network architecture for automated recognition of intracellular malaria. Retrieved from http://kosmi. snubi.org/2003_fall/APAMI_CJKMI/ O3-3-020-Premaratne-0731.pdf

Vvan der Laan, M. \& Pollard, K. (2003). Hybrid clustering of gene expression data with visualization and the bootstrap. Journal of Statistical 
Planning and Inference, 117(2). 275-303. in the determination of the Ki-67 Kim, Y., \& Romeike, B. (2006). labeling index in meningiomas. Clinical Automated nuclear segmentation Neuropathology, Vol 25(2), 67-73. 


\section{Currículum vitae}

\section{Leonardo Yunda, Ph.D (c).}

Dean of Engineering School (Universidad Santiago de Cali, USC) and Director-Researcher in T@lebio, a Research Group within USC. Electronic Engineer (2000) and Master in Engineering (2006) from Universidad del Valle; Master in Telematics Engineering (2010) y Ph.D candidate in Telematics Engineering from Universidad de Vigo (España). Since 2001 until 2004 he worked as a researcher and development engineer in the Fraunhofer-IBMT

Technology Center (Hialeah, FL), a branch of the Fraunhofer Institute for Biomedical Technology in St. Ingbert, Germany. His mayor areas of interest are Telemedicine and Digital Signal Processing.

\section{Jorge Millán, Ph.D.}

Physics (Universidad del Valle, 1988) with a Master degree in Physics (Universidad de Puerto Rico, 1993) and Electric Engineering (Pennsylvania University, 2000), and a Ph.D., in Biomedical Engineering (Universidad de Miami, 2006). Executive Director from Sigma Biomedical (Hialeah, FL), a contract research and development biomedical

engineering company established to serve the scientific, engineering and regulatory needs of the biomedical sector, who provides custom solutions by a synergy of advanced instrumentation, biomedical signal processing, health telematics, medical imaging, medical device testing, software verification and validation, software and hardware development, mechanical engineering as well as medical device regulatory expertise.

\section{Andrés Alarcon Ramirez, M.Sc.}

Electronic Engineer with a Master degree in Electronic Engineering (both from Universidad del Valle), presently linked to the Laboratory for Applied Remote Sensing and Image Processing (LARSIP), a multidisciplinary laboratory dedicated to the research and implementation of Remote Sensing, Hyperspectral Image Processing, Signal and Image

Processing, Geographical Information Systems (GIS),Emergency Response Systems, Global Positioning Systems (GPS) technologies, Applied Electromagnetics and Bio-Optics, located within the Department of Electrical and Computer Engineering at the University of Puerto Rico, Mayagüez Campus. His mayor areas of interest are Communication's Networks and Digital Signal Processing. 